\title{
Decoherence, Time Scales and Pointer States
}

\author{
Tabish Quresh:* \\ Centre for Theoretical Physics, Jamia Millia Islamia, New Delhi-110025, INDIA.
}

\begin{abstract}
Certain issues regarding the time-scales over which environment-induced decoherence occurs, and the nature of emergent pointer states, are discussed. A model system, namely, a Stern-Gerlach setup coupled to a quantum mechanical "heat-bath" is studied. The emergent pointer states for this system are obtained, which are different from those discussed in the literature. It is pointed out that this difference is due to some confusion regarding the decoherence time-scale, which is clarified here.

PACS numbers: 03.65.Yz
\end{abstract}

\section{INTRODUCTION}

The emergence of classical world from quantum mechanics is a subject which is poorly understood. While the theories decribing the classical and quantum objects are well established, the border between the two still remains hazy for many. There have been many attempts towards resolving this issue. Most of them either modify quantum mechanics, e.g., the so called GRW proposal [1], or introduce certain hidden variables, e.g., the deBroglieBohm approach.

One approach which tries to tackle the problem using only conventional quantum mechanics forms the basis of the decoherence program, initiated by Zeh [2], and later extensively developed by Zurek and collaborators [3]. Decoherence approach is based on the observation that macroscopic objects can never be considered approximately isolated. They are always interacting weakly with various external degrees of freedom, collectively referred to as the environment, which are normally ignored. The interaction with the environment leads to, argues Zeh, a dephasing of the system. This leads to certain phase relations between the macroscopically separated parts of the wave-function, getting lost. This information is not really lost, but leaks away almost irreversibly into the infinitely large number of degrees of freedom of the environment. The result is that the initial pure state density matrix of the system now appears to be a mixed state density matrix, if one decides not to look at the environment variables, and focuses attention only on the system of interest. It should be noted that while the time evolution of the system plus environment is completely unitary, the system by itself appears to undergo a nonunitary change. This process leads to the appearance of a classically interpretable probability distribution, and is believed to describe the emergence of the classical world.

A prototype model that one studies in this context is that of a free particle coupled to a "heat-bath" of an infinite number of independent harmonic oscillators [4 6 ]. This model becomes tractable in the so-called Markovian approximation. In this limit the time evolution of the

*Email: tabish@ctp-jamia.res.in density matrix is governed by a master equation. Zurek did an approximate study of this master equation and showed that quantum coherence between two pieces of the wave-function, spatially separated by a distance $\Delta x$, is destroyed over a time-scale given by

$$
\tau_{D} \approx \gamma^{-1} \frac{\hbar^{2}}{2 m k_{B} T(\Delta x)^{2}}
$$

where $\gamma^{-1}$ is the relaxation time of the system, $k_{B} T$ is the thermal energy of the heat-bath, assumed to be in equilibrium, and $m$ is the mass of the particle. As one can see, for a particle of mass $1 \mathrm{~g}$, interacting with a heatbath at $300 \mathrm{~K}$, the quantum coherence over a distance of $1 \mathrm{~cm}$ is destroyed in a time which is $10^{-40}$ times the relaxation time.

Zurek also concluded from this analysis that the environment selects position as a preferred basis in which the density matrix of the particle becomes diagonal. The states constituting this basis, selected by environment induced decoherence, have come to be known as preferred states or pointer states. The nature of pointer states has also been a subject of much research attention [8, 9].

Recently, related models have been studied in a more rigorous manner to understand the detailed working of decoherence $10-13$. Interestingly, the conclusions of these studies are at variance with Zurek's approximate calculation.

The purpose of this investigation is to have a fresh look at one model and get a clear picture regarding the decoherence time and pointer states.

\section{WHAT TO EXPECT?}

Let us first discuss what one should expect as a result of decoherence, if one believes that it leads to the emergence of classicality. We should of course expect that there will be no Schrödinger's cats hanging around, that is, there should be no superpositions of spatially separated states. Zurek's analysis achieves that as he shows that quantum coherence over macroscopic distances dies out very very fast. But that alone doesn't rule out the existence of superpositions of macrosopically distinct momentum. 
Classically we should also anticipate that quantum coherence will be destroyed over macroscopic distances in the momentum space too. Then classical particles will have macroscopically well-defined position, as well as momentum, and their dynamics could be characterized by paths. Infact, macroscopic superpositions of no classical observable should exist. Such should be the nature of the pointer states that emerge. In this light, Zurek's claim of position emerging as the preferred basis [7] and that of some other works 10 12 where momentum seems to emerge as pointer states, do not seem to satisfy the criterion of "classicality". On the other hand, in the work of Zurek and Paz [15] and also of Venugopalan [13] coherent states seem to emerge as pointer states for a harmonic oscillator, which nicely satisfy the "classicality" criterion.

We should also expect that full classical dynamics will emerge from decoherence, which means that the effect of interaction with the environment will have negligibly small influence on the dynamics. It is worth pointing out that the same models have been in use for studying quantum dissipation [ [5, [] , where one studies the influence of a heat-bath on the dynamics of a quantum system. Since we are assuming that our system of interest is classically an isolated system, no dissipative effect should be observable even if decoherence is strong. This seems like a stringent condition, but it will turn out that this is not so.

Another thing one might expect is that decoherence would be faster if the interaction with the environment is stronger. Zurek's expression for decoherence time $\tau_{D} \approx \gamma^{-1} \frac{\hbar^{2}}{2 m k_{B} T(\Delta x)^{2}}$ seems to be in agreement with this line of thought. The decoherence time here is inversely proportional to $\gamma$, which is also a measure of the strength of interaction with the environment.

\section{THE STERN-GERLACH SETUP}

In the following, we look at a model, which has already been studied before, and try to understand how decoherence works.

Consider a particle of mass $m$ and spin $\frac{1}{2}$, moving along the y-axis, and passing through a magnetic field along the z-axis, which is inhomogeneous along the $\mathrm{x}$ axis. This model typically describes a Stern-Gerlach experiment, with a Hamiltonian

$$
H_{0}=\frac{p^{2}}{2 m}+\epsilon x \sigma_{z}
$$

where $x$ and $p$ denote the position and momentum operators of the particle, and $\sigma_{z}$ is the Pauli spin matrix, denoting the z-component of the spin operator, multiplied by 2 .

In addition, the particle is assumed to interact with a set of infinite number of harmonic oscillators, through the interaction

$$
H_{I}=\sum_{j} \frac{P_{j}^{2}}{2 m_{j}}+\frac{1}{2} m_{j} w_{j}^{2}\left(X_{j}-\frac{g_{j} x}{m_{j} \omega_{j}^{2}}\right)^{2},
$$

which is just the Hamiltonian for a set of independent but shifted harmonic oscillators, with the shift proportional to the position of the particle. This in effect, represents a coordinate-coordinate coupling of the particle with the oscillators, with $g_{j}$ as coupling constants. The aim is to study the reduced density matrix of the system by tracing over the oscillator degrees of freedom. The offdiagonal matrix elements represent presence of quantum coherence in the system.

It turns out that in order to analyze the influence of the environment consisting of harmonic oscillators on the dynamics of the particle, one does not need individual knowledge of $g_{j} \mathrm{~s}, m_{j} \mathrm{~s}$ and $\omega_{j} \mathrm{~s}$. One only requires them in a particular combination as they appear in the so-called spectral density function :

$$
J(\omega)=\sum_{j=0}^{\infty} \frac{g_{j}^{2}}{m_{j} \omega_{j}} \delta\left(\omega-\omega_{j}\right) .
$$

In order to describe the dissipative behavior that goes to the correct classical limit, one assumes a continuous spectrum of frequencies in the bath, with the spectral density taking the "Ohmic" form where $J(\omega)$ is linear in $\omega$ for small frequencies:

$$
J(\omega)=\gamma \omega e^{-\omega / \omega_{c}},
$$

where $\omega_{c}$ is a large cut-off frequency.

Clearly, $\gamma$ is now a measure of the strength of the coupling between the particle and the environment. All the $g_{j}$ s going to zero would amount to $\gamma$ going to zero, which would represent a decoupled system and heat-bath.

This model has been studied before by the pathintegral technique [10] and the master equation technique [11]. In both the works, the conclusion is that the offdiagonal elements (in the spin-space) of the density matrix decay over a time-scale

$$
t_{s}=\left(\frac{3 \hbar^{2} m \gamma}{2 \epsilon^{2} k_{B} T}\right)^{1 / 3}
$$

It is also concluded that the spin-diagonal components of the density matrix become diagonal in the momentum space over a decoherence time-scale

$$
\tau_{D}=\frac{m \gamma}{2 k_{B} T(\Delta p)^{2}},
$$

where $\Delta p$ is the spread in the momentum space.

Let us look at the the reduced density matrix for the particle more carefully. For simplicity, let us assume that the particle started as a Gaussian wave-packet of width $\sigma$, with mean momentum zero, meaning the particle has 
no motion along $\mathrm{x}$-axis when it travels along $\mathrm{y}$-axis. The spin diagonal elements of the reduced density matrix can be written as 11]

$$
\begin{aligned}
\rho_{ \pm}(R, r, t) & =2 \sqrt{\frac{\pi}{M(\tau)}} \exp \left\{-\left(\frac{e^{-2 \tau}}{4 \sigma^{2}}+\frac{D\left(1-e^{-2 \tau}\right)}{8 \hbar^{2} \gamma}\right) r^{2}\right. \\
& \mp \frac{i \epsilon}{\hbar \gamma}\left(1-e^{-\tau}\right) r \\
& -\frac{1}{M(\tau)}\left(R \pm \frac{\epsilon}{m \gamma^{2}}\left(1-e^{-\tau}-\tau\right)\right. \\
& \left.\left.-\frac{i \hbar r}{2 \sigma^{2} m \gamma} e^{-\tau}\left(1-e^{-\tau}\right)+\frac{i D r}{4 m \gamma^{2} \hbar}\left(1-e^{-\tau}\right)^{2}\right)^{2}\right\}
\end{aligned}
$$

of it, look like

$$
\begin{aligned}
\rho_{ \pm}(R, r, t) \approx & 2 \sqrt{\frac{\pi}{\sigma^{2}}} \exp \left(-\frac{2 m \gamma k_{B} T\left(x-x^{\prime}\right)^{2}}{\hbar} t\right) \\
& \times \exp \left(\mp \frac{i \epsilon x t}{\hbar}-\frac{1}{2 \sigma^{2}}\left(x \mp \frac{\epsilon t^{2}}{2 m}\right)^{2}\right) \\
& \times \exp \left( \pm \frac{i \epsilon x^{\prime} t}{\hbar}-\frac{1}{2 \sigma^{2}}\left(x^{\prime} \mp \frac{\epsilon t^{2}}{2 m}\right)^{2}\right) .
\end{aligned}
$$

Barring the exponential time-decay term, this represents a pure state density matrix for a Gaussian wave-packet, $\int$ centered at $x= \pm \frac{\epsilon t^{2}}{2 m}$, with a mean momentum $\bar{p}= \pm \epsilon t$. This has a nice classical interpretation - with an accelera(ton $\epsilon / m$, after a time $t$, the particle would have travelled where $\tau=\gamma t, D=8 m \gamma k_{B} T, r=x-x^{\prime}, R=\frac{x+x^{\prime}}{2}$ and

$M(\tau)=\sigma^{2}+\frac{\hbar^{2}\left(1-e^{-\tau}\right)^{2}}{\sigma^{2} m^{2} \gamma^{2}}+\frac{D}{2 m^{2} \gamma^{3}}\left(2 \tau-3+4 e^{-\tau}-e^{-2 \tau}\right)$.

Now, this is an exact solution of the master equation, and needs to be looked at in some appropriate limit which satisfy the criteria we spelt out in section II. In order that dissipative effects do not show up in the dynamics, the relaxation time $\gamma^{-1}$ must be much much larger than any other time of interest, i.e., $\gamma t \ll 1$. We could, for example, easily consider $\gamma^{-1}$ to be 100 years, and then look at the dynamics for any long time meaningful in the lab. So, for $\gamma t \ll 1$, the expression for the spin-diagonal components of the density matrix takes the approximate form

$$
\begin{aligned}
& \rho_{ \pm}(R, r, t) \approx \\
& \quad 2 \sqrt{\frac{\pi}{\sigma^{2}}} \exp \left\{-\frac{2 m \gamma k_{B} T\left(x-x^{\prime}\right)^{2}}{\hbar} t\right) \\
& \quad \times \exp \left(\mp \frac{i \epsilon x t}{\hbar}-\frac{1}{2 \sigma^{2}}\left(x \mp \frac{\epsilon t^{2}}{2 m}-\frac{i \hbar r t}{2 \sigma^{2} m}+\frac{i D r t^{2}}{4 m \hbar}\right)^{2}\right) \\
& \quad \times \exp \left( \pm \frac{i \epsilon x^{\prime} t}{\hbar}-\frac{1}{2 \sigma^{2}}\left(x^{\prime} \mp \frac{\epsilon t^{2}}{2 m}-\frac{i \hbar r t}{2 \sigma^{2} m}+\frac{i D r t^{2}}{4 m \hbar}\right)^{2}\right) .
\end{aligned}
$$

One can see that there is an exponential decay term which destroys parts with finite $\left(x-x^{\prime}\right)^{2}$, and leads to decoherence. And this happens over a time-scale

$$
\tau_{D}=\frac{\hbar^{2}}{2 m \gamma k_{B} T\left(x-x^{\prime}\right)^{2}} .
$$

One can see that Zurek's approximate treatment is in agreement with this, and so is the path-integral calculation of Banerjee and Ghosh [10]. The imaginary terms in the arguments of the two Gaussians depend on $\left(x-x^{\prime}\right)$, and hence become inconsequential because of fast destruction of terms involving $\left(x-x^{\prime}\right)$. If one ignores them, the density matrix, rather the spin diagonal components a distance $\frac{\epsilon t^{2}}{2 m}$, and would have acquired a momentum $\epsilon t$. Thus one sees that although there is fast decoherence, the classical dynamics remains intact. So, the spin states are correlated with the wave-packets moving in different directions.

One might be tempted to conclude from the preceding analysis that position basis constitutes the pointer states. But before that we should also look at what the spin-diagonal density matrix looks like in the momentum basis. The spin diagonal elements of the reduced density matrix can be written, in the momentum basis, as [1]

$$
\begin{aligned}
& \rho_{ \pm}(Q, q, t)= \\
& \quad 2 \sqrt{\frac{\pi}{N(\tau)}} \exp \left\{-\frac{1}{N(\tau)}\left(q \mp \frac{\epsilon}{\hbar \gamma}\left(1-e^{-\tau}\right)\right.\right. \\
& \left.+\frac{i \hbar Q}{2 \sigma^{2} m \gamma} e^{-\tau}\left(1-e^{-\tau}\right)-\frac{i D Q}{4 m \gamma^{2} \hbar}\left(1-e^{-\tau}\right)^{2}\right)^{2} \\
& \quad-\left(\frac{\hbar^{2}}{4 \sigma^{2} m^{2} \gamma^{2}}\left(1-e^{-\tau}\right)^{2}+\frac{\sigma^{2}}{4}\right. \\
& \left.+\frac{D}{2 m^{2} \gamma^{3}}\left(2 \tau-3+4 e^{-\tau}-e^{-2 \tau}\right)\right) Q^{2} \\
& \left.+\left(\mp \frac{i \epsilon \tau}{m \gamma^{2}} \pm \frac{i \epsilon}{m \gamma^{2}}\left(1-e^{-\tau}\right)^{2}\right) Q\right\}
\end{aligned}
$$

where

$$
N(\tau)=\frac{D}{2 \hbar^{2} \gamma}\left(1-e^{-2 \tau}\right)+\frac{1}{\sigma^{2}} e^{-2 \tau} .
$$

Again, this should be looked at in the meaningful limit $\gamma t \ll 1$. In this limit, it takes the form,

$$
\begin{aligned}
\rho_{ \pm}(Q, q, t) \approx & 2 \sigma \sqrt{\pi} \exp \left(-\frac{2 \gamma k_{B} T\left(p-p^{\prime}\right)^{2} t^{3}}{3 m \hbar^{2}}\right) \\
& \times \exp \left(\mp \frac{i \epsilon t^{2} p}{2 m \hbar}-\frac{\sigma^{2}}{2 \hbar^{2}}(p \mp \epsilon t)^{2}\right) \\
& \times \exp \left( \pm \frac{i \epsilon t^{2} p^{\prime}}{2 m \hbar}-\frac{\sigma^{2}}{2 \hbar^{2}}\left(p^{\prime} \mp \epsilon t\right)^{2}\right)
\end{aligned}
$$

where we have assumed that because of the exponential time decay due to the first term, the imaginary terms involving $Q$ in the exponent of (13), can be ignored. 
Here, one can see that the momentum off-diagonal elements decay with time in a way that is not strictly exponential because of the $t^{3}$ in the exponent, instead of just $t$. Nevertheless, one can extract a decay time scale given by

$$
\tau_{D}=\left(\frac{3 m \hbar^{2}}{2 \gamma k_{B} T\left(p-p^{\prime}\right)^{2}}\right)^{1 / 3}
$$

Barring the first term, the rest of the expression represents a pure state density matrix for a momentum wavepacket, centered at $p= \pm \epsilon t$, and with a mean position $\pm \frac{\epsilon t^{2}}{2 m}$. This again has a nice classical interpretation of a particle having travelled a distance $\pm \frac{\epsilon t^{2}}{2 m}$, and having acquired a momentum $\pm \epsilon t$, because of an acceleration $\pm \epsilon / m$ due to the Stern-Gerlach field.

Thus the environment selects out Gaussian wavepackets approximately localized both in position and momentum, as pointer states. So, the "classicality" criterion, discussed in section II is satisfied here. This result is in agreement with the earlier work by Eisert where it is shown that decoherence to Gaussian states is generic for free particles coupled to heat-bath [16]. Here we see that even for the Stern-Gerlach setup, Gaussian states emerge as pointer states, although with a centre that moves according to classical dynamics. So, the undamped Newtonian dynamics is recovered. Thus, the role of the environment is only to localize spatially extended states. The Newtonain dynamics, which is guaranteed by the Ehrenfest theorem, emerges naturally. With (approximately) well-defined position and momentum, one can now talk of classical paths. So, because of decoherence, quantum particles acquire trajectories.

Earlier works [10, 11] conclude that the pointer states for this problem are momentum states, and the decoherence time is given by (7). Expression (7) cannot denote the true decoherence time, because it depends inversely on the relaxation time of the particle. This would mean, if the interaction with the environment, parametrized by $\gamma$ is decreased, the decoherence should be faster. That cannot obviously be true. The catch lies in the fact that in earlier works [10, 11], the authors used the limit $\gamma t \gg 1$, which takes one to the regime where there is not just decoherence, but also dissipation. Decoherence happens very fast, in the time within the limit $\gamma t \ll 1$. So, it is meaningful to use this limit to study decoherence.

In the limit $\gamma t \gg 1$ dissipative effects overshadow the effect of decoherence in the dynamics. This limit is physically meaningful in studying the damped dynamics of quantum particles. The particle moving under a potential, loses energy and ultimately thermalizes with the heat-bath, ending in a meandering trajectory. For example, a quantum harmonic oscillator, in this limit would be damped to the extent that it comes to its mean position and executes thermal motion.

It should be pointed out that if pointer states were either momentum states as claimed in [10,11], or position states as claimed by Zurek [7], the density matrix would remain non-diagonal in the conjugate variable, and the classicality criteria won't be satisfied. In our analysis, Gaussian states with moving centers, emerge as natural pointer states. They look closer to classical states, and also yield undamped Newtonian dynamics.

\section{CONCLUSIONS}

In this paper, we have clarified certain issues regarding the time-scales over which environment-induced decoherence occurs, and the nature of emergent pointer states. A model system, namely, the Stern-Gerlach setup, coupled to a quantum mechanical "heat-bath" has been studied. The emergent pointer states are Gaussian states which evolve following Newtonian dynamics.
[1] G. C. Ghirardi, A. Rimini, and T. Weber, Phys. Rev. D 34, 470 (1986).

[2] H.D. Zeh, Found. Phys. 1, 69 (1970); E. Joos and H.D. Zeh, Z. Phys. D 59, 223 (1985).

For a recent review, see Decoherence and the appearance of a classical world in quantum theory, eds. Guilini et al. (Springer) (1996).

[3] Decoherence, einselection, and the quantum origins of the classical, W. H. Zurek, Rev. Mod. Phys. 75, 715-775 (2003).

[4] R.P. Feynman and F.L. Vernon, Ann. Phys.(N.Y.) 24, 118 (1963).

[5] H. Dekker, Phys. Rep. 80, 1 (1981).

[6] A.O. Caldeira and A.J. Legget, Physica A 121, 587 (1983).

[7] Decoherence and the transition from quantum to classical, W. H. Zurek, Physics Today 44 (10), 36 (1991).

[8] Predictability sieve, pointer states, and the classicality of quantum trajectories, D.A.R. Dalvit, J. Dziarmaga, W. H. Zurek, Phys. Rev. A 72, 062101 (2005).

[9] Environment-induced super selection without pointer states, C. Gogolin, Phys. Rev. E 81, 051127 (2010).

[10] Quantum theory of a Stern-Gerlach system in contact with a linearly dissipative environment, S. Banerjee and R. Ghosh, Phys. Rev. A 62, 042105 (2000).

[11] Environment-induced decoherence I: The Stern-Gerlach measurement, A. Venugopalan, D. Kumar, R. Ghosh, Physica 220, 563 (1995).

[12] Preferred basis in a measurement process, A. Venugopalan, Phys. Rev. A 50, 2742 (1994).

[13] Pointer states via decoherence in a quantum measurement, A. Venugopalan, Phys. Rev. A 61, 012102 (1999).

[14] W. H. Zurek, S. Habib and J. P. Paz, Phys. Rev. Lett. 70, 1187 (1993).

[15] Quantum limits of decoherence: environment induced super-selection of energy eigenstates, J. P. Paz and W. 
H. Zurek, Phys. Rev. Lett. 82, 5181 (1999).

210401 (2004).

[16] Exact Decoherence to Pointer States in Free Open Quantum Systems is Universal, J. Eisert, Phys. Rev. Lett. 92, 